\title{
USER RECOMMENDATION ALGORITHM IN SOCIAL TAGGING SYSTEM BASED ON HYBRID USER TRUST
}

\author{
Norwati Mustapha, Wong Pei Voon and Nasir Sulaiman \\ Department of Computer Science, Faculty of Computer Science and Information Technology, \\ University Putra Malaysia, Serdang, Malaysia
}

Received 2013-02-21, Revised 2013-06-13; Accepted 2013-07-05

\begin{abstract}
With the rapid growth of web 2.0 technologies, tagging become much more important today to facilitate personal organization and also provide a possibility for users to search information or discover new things with Collaborative Tagging Systems. However, the simplistic and user-centered design of this kind of systems cause the task of finding personally interesting users is becoming quite out of reach for the common user. Collaborative Filtering (CF) seems to be the most popular technique in recommender systems to deal with information overload issue but CF suffers from accuracy limitation. This is because CF always been attack by malicious users that will make it suffers in finding the truly interesting users. With this problem in mind, this study proposes a hybrid User Trust method to enhance CF in order to increase accuracy of user recommendation in social tagging system. This method is a combination of developing trust network based on user interest similarity and trust network from social network analysis. The user interest similarity is derived from personalized user tagging information. The hybrid User Trust method is able to find the most trusted users and selected as neighbours to generate recommendations. Experimental results show that the hybrid method outperforms the traditional CF algorithm. In addition, it indicated that the hybrid method give more accurate recommendation than the existing $\mathrm{CF}$ based on user trust.
\end{abstract}

Keywords: User Trust, Tag, Collaborative Filtering

\section{INTRODUCTION}

Collaborative Tagging Systems (Begelman et al., 2006; Gemmell et al., 2009a; 2009b; Hotho et al., 2006; Shepitsen et al., 2008) allow users to label digital resources using free-form of keywords (tags). The simplistic and the user-centered design of this kind of systems have encouraged many Web users to annotate their data using tags (Begelman et al., 2006; Gemmell et al., 2009a; 2009b; Hotho et al., 2006; Shepitsen et al., 2008). Collaborative Tagging Systems allow users to explore other users' bookmarks via the keywords and tracking users who bookmarked pages that you considered interesting (Begelman et al., 2006; Gemmell et al., 2009a; 2009b; Hotho et al., 2006; Shepitsen et al., 2008). Nonetheless, users will find it hard to search other users with similar interest within a social tagging system that have hundreds of thousands of user with users racking up tens of thousands of bookmarks.

CF algorithm seems to be the most popular technique in recommender systems (Konstan et al., 1997; Resnick et al., 1994; Sarwar et al., 2000; Tso-Sutter et al., 2008) to deal with information overload issue. However, traditional CF algorithm focuses only on similar users' opinions which express in ratings and do not consider the actual content of the items, which affected the quality of the recommendation. To improve recommendation quality, metadata such as content information in items and tags have been typically used as additional knowledge.

In the past few years, the dramatic expanding of Web 2.0 Web sites and applications poses new challenges for traditional CF recommender systems. Traditional CF University Putra Malaysia, Serdang, Malaysia 
recommender systems only generate recommendations based on similar users' opinions and ignore user trust relationships among users (Golbeck, 2006; Liu and Lee, 2010; Yubo et al., 2010). In daily life, when people seek advice from peers, they consider their past interaction history to locate the right peer, or if advice is received, they utilize these past interactions to judge the advice quality (Bhuiyan et al., 2010; Golbeck, 2006; Liu and Lee, 2010; Tyler and Zhang, 2008; Yubo et al., 2010). Furthermore, users would prefer to receive recommendations from people that they trust.

Recently, based on the intuition that users' trust relations can be employed to enhance traditional $\mathrm{CF}$ recommender systems, a few trust recommendation methods have been proposed (Bhuiyan et al., 2010; Golbeck, 2006; Liu and Lee, 2010; Tyler and Zhang, 2008; Yubo et al., 2010). These methods utilize the inferred implicit or observed explicit trust information to further improve the traditional $\mathrm{CF}$ recommender systems.

However, CF recommender systems are vulnerable to attack by malicious users. The attacker creating a user profile that mirrors the ratings that some target user has made. The system will then employ the attacker's (very similar) profile when making recommendations for the target user and as a result every additional item that the attacker rates highly will be recommended to the target user. The malicious users affect the trustworthiness of $\mathrm{CF}$ recommendation that cause the level of existing recommendation accuracy to date is still at unsatisfactory level among the users.

In this study, we propose a hybrid User Trust method for user recommendation to allow users to easily find other users with similar interest in social tagging system. This method is a combination of developing trust network based on user interest similarity and trust network from social network analysis. The user interest similarity is derived from personalized user tagging information. The hybrid User Trust method is able to find the most trusted users and selected them as neighbours to generate recommendations.

\section{MATERIALS AND METHODS}

The aim of our approach is to provide a hybrid User Trust method for user recommendation, which enable users to easily find other users with similar interest within a particular social tagging system. The overview of user recommendation based on hybrid User Trust method is illustrated in Fig. 1.

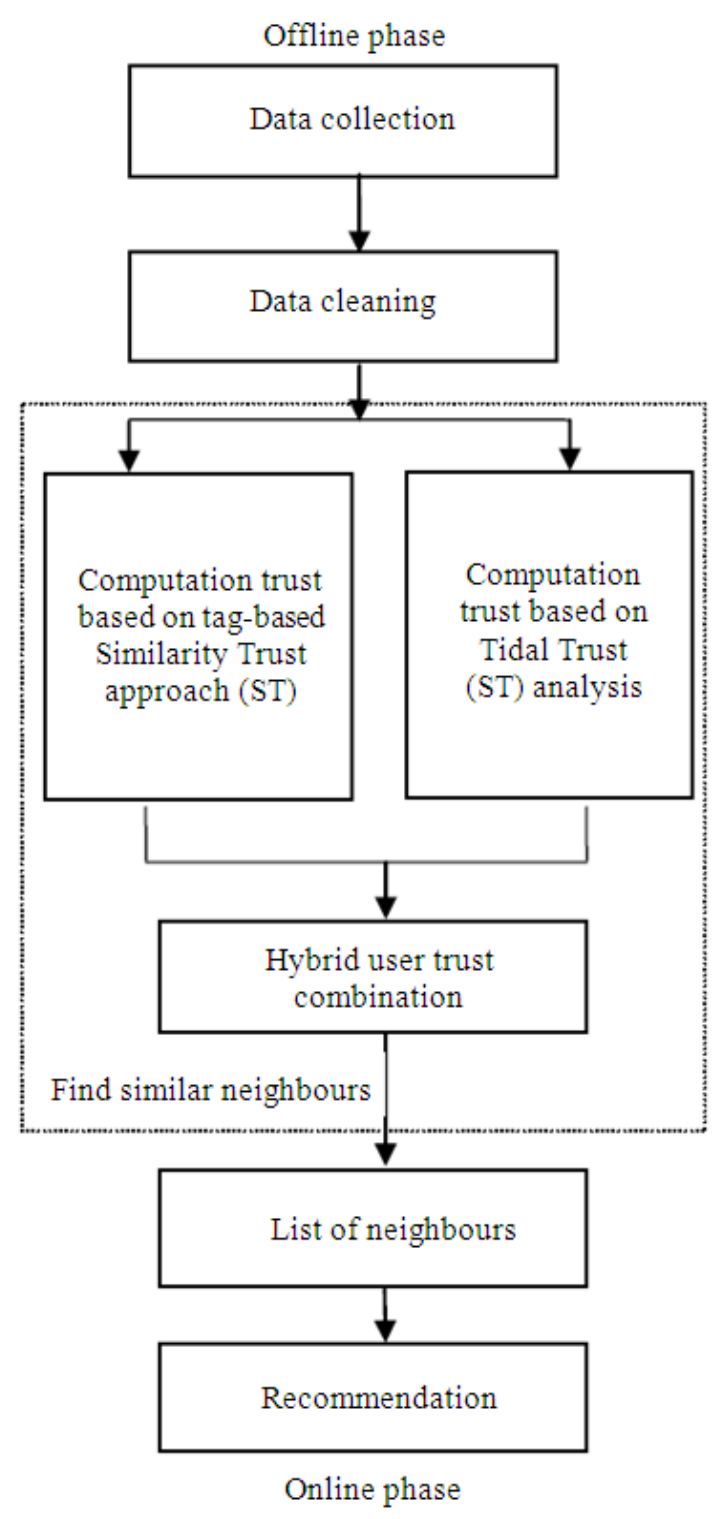

Fig. 1. User recommendation based on hybrid user trust

Based on Fig. 1, the model can be partitioned into two main phases: Offline phase and online phase. Despite the separation of process in the model, the online phase is strongly affected by the offline phase.

\subsection{Offline Phase of Hybrid User Trust for User Recommendation}

There are three main modules consisted in this phase, which are data collection, data cleaning and finding similar neighbours. 


\subsection{Data Collection}

In the first module, data are collected in the form of about users' tags and their social network relationships from a social tagging Web site called Del.icio.us. This dataset comes from the first and the most popular collaborative tagging system that has been in existence since the end of 2003. The system allows users to tag bookmark (URLs) with personal keywords. It also helps to collect more input data for the purpose of user profiling and personalization (Noll and Meinel, 2007).

In addition, users may add other users who share similar interest to their personal network. Users are informed with the latest interesting resources added by people from his or her network. Users are also informed with the list of users who have added him or her into their personal network including a list of fans.

In specific, the dataset del.icio.us (http://www.delicious.com/) contains users' tagging activities, network information and fan information. The dataset is provided by Chinese University of Hong Kong and is used in Zhou et al. (2010) research.

\subsection{Data Cleaning}

In the original Del.icio.us dataset, not all the entries are valid for user recommendation. Therefore, data cleaning is used to eliminate the irrelevant entries from the dataset, which includes: time, hashcode and people for how many users have bookmarked this URL.

\subsection{Find Similar Neighbours}

The user-based collaborative filtering approach is a traditional method that consists of two steps. In the first step, this approach finds the similar neighbours based on the overlap of previous ratings data and in the second step, it computes the top-N users for target user (Bennett and Lanning, 2007; Konstan et al., 1997; Linden et al., 2003; Resnick et al., 1994; Sarwar et al., 2000).

As for the first part of this experiment, which is finding the neighbours, we propose to use trust as an alternative method in the absence of explicit rating data to find the neighbours and replace the first step of traditional $\mathrm{CF}$ method where it finds the neighbours based on over-lapped or common previous ratings data. It is because we believe the trustworthiness between users is useful for making recommendation. However, the trust information is not always available and even available, it may change over time. In this research, we propose two different methods to construct the trustworthiness between users based on users' online information and online behavior to reduce trustworthiness attack by malicious users.

The first method is by using users' interest similarity derived from user's personalized tagging information in order to form the trust network among the users irrespective of their personal relationship. Tagging is very useful for users to figure out other users with similar interests within a given category. Users with similar interests might post similar tags and similar resources might have similar tags posted to them. This method is based on tag-based Similarity Trust (ST) method as proposed by Bhuiyan et al. (2010). The second method is by using user's network to infer the trust network based on Tidal Trust (TT) proposed by Golbeck (2006). We used TT because it is simple and it low complexity allows high scalability in application. Finally, we sum up the values from both trust networks from the two separate models to find the neighbours and to make automated recommendations.

\subsection{Tag-Based Similarity Trust Approach (ST)}

In this research, we use ST (Bhuiyan et al., 2010) to automatically construct the trustworthiness among users based on users' online information and online behavior. We define some concepts used in this research as follows.

\section{Users (U)}

$\mathrm{U}=\left\{\mathrm{u}_{1}, \mathrm{u}_{2}, \ldots, \mathrm{u}_{|\mathrm{U}|}\right\}$ contains all users in an online community who are using tags to label and organize items.

\section{Items (i.e., Products, Resources)}

$\mathrm{P}=\left\{\mathrm{p}_{1}, \mathrm{p}_{2}, \ldots, \mathrm{p}_{|\mathrm{P}|}\right\}$ contains everything that is being tagged by users in U. Items could be any type of online information resources or products in an online community such as Web pages, videos, music tracks, photos, academic papers, documents or books.

\section{Tags}

$\mathrm{T}\left\{\mathrm{t}_{1}, \mathrm{t}_{2}, \ldots, \mathrm{t}_{\mathrm{T} \mid}\right\}$ contains all tags used by users in $\mathrm{U}$. A tag is an arbitrary word that users use to label or collect items.

Nonetheless, the representation of tags is often using free-style vocabulary that users use to annotate their items. The freedom afforded by the users comes at a cost, which is uncontrolled vocabulary that results in tag ambiguity (Bhuiyan et al., 2010; Gemmell et al., 2009a; 2009b; Hotho et al., 2006; Jaschke et al., 2007). Moreover, the tags are usually short and containing only one or two words, which make it even harder to truly get the semantic meaning of the tags (Bhuiyan et al., 2010). 
To solve this problem, we apply the approach as proposed by Bhuiyan et al. (2010) to extract the semantic meaning of a tag based on the title of the items in that tag. For each item, Bhuiyan et al. (2010) assume that there is a set of keywords or topics that describe the content of the item. This assumption is often true in reality. For most items, normally when there is an item, there will be a title along the item. From the item title, by using the tf-idf weighing scheme, we can generate a set of keywords in order to represent the content of the item.

After the keyword extraction process, trust values among users are computed using conditional probability.

Given user $\mathrm{u}_{\mathrm{i}} \in \mathrm{U}$, let $\mathrm{T}_{\mathrm{i}}=\left\{\mathrm{t}_{\mathrm{i} 1}, \ldots, \mathrm{t}_{\mathrm{i} l}\right\} \subseteq \mathrm{T}$ be a set of tags that are used by $u_{i}$. For each tag $t_{i j} \in T_{i}$, by using tf-idf weighing scheme, from the title of the items in $t_{i j}$, we can generate a set of frequent keywords denoted as $\mathrm{W}_{\mathrm{ij}}\left\{\mathrm{w}_{1}, \ldots, \mathrm{w}_{\mathrm{n}}\right\}$ to represent the semantic meaning of the tag. The weight of the keywords, denoted as $\mathrm{v}_{\mathrm{ij}}=$ $\left\langle\mathrm{f}_{1}, \ldots, \mathrm{f}_{\mathrm{n}}\right\rangle$ where $\mathrm{f}_{\mathrm{k}}$ is the frequency of the $\mathrm{k}^{\text {th }}$ keyword, measures the strength of each keyword in tag $t_{i j}$ to represent the meaning of the tag. Also, the vector $\mathrm{v}_{\mathrm{ij}}$ can be used to calculate the similarity of two tags in terms of their semantic meaning $\forall \mathrm{u}_{\mathrm{i}}, \mathrm{u}_{\mathrm{j}} \in \mathrm{U}$ and let $\mathrm{T}_{\mathrm{i}}=\left\{\mathrm{t}_{\mathrm{i} 1}, \ldots, \mathrm{t}_{\mathrm{il}}\right\}$, $\mathrm{T}_{\mathrm{j}}=\left\{\mathrm{t}_{\mathrm{j} 1}, \ldots, \mathrm{t}_{\mathrm{j} 1}\right\} \subseteq \mathrm{T}$ be the set of tags that are used by user $\mathrm{u}_{\mathrm{i}}$ and $\mathrm{u}_{\mathrm{i}}$ respectively.

Corresponding to $\mathrm{T}_{\mathrm{i}}$ and $\mathrm{T}_{\mathrm{j}}, \mathrm{W}_{\mathrm{i}}\left\{\mathrm{w}_{\mathrm{i} 1}, \ldots, \mathrm{W}_{\mathrm{in}}\right\}$ and $\mathrm{W}_{\mathrm{j}}\left\{\mathrm{w}_{\mathrm{j} 1}, \ldots, \mathrm{w}_{\mathrm{jm}}\right\}$ are the collection of keyword sets for the tags in $\mathrm{T}_{\mathrm{i}}$ and $\mathrm{T}_{\mathrm{j}}$ respectively and $\mathrm{V}_{\mathrm{i}}\left\{\mathrm{v}_{\mathrm{i} 1}, \ldots, \mathrm{v}_{\mathrm{im}}\right\}$ and $V_{j}\left\{v_{j 1}, \ldots, v_{j m}\right\}$ are the corresponding vectors of weighted keyword. For example, $w_{j 1}$ is the set of keywords derived from the items title in tag $t_{j 1}$ and $v_{j 1}$ is the weight of the keywords in $\mathrm{w}_{\mathrm{j} 1}$. Let $\operatorname{sim}\left(\mathrm{v}_{\mathrm{jp}}, \mathrm{v}_{\mathrm{jq}}\right)$ be the similarity between $\mathrm{v}_{\mathrm{jp}}$ and $\mathrm{v}_{\mathrm{jq}}$, if $\operatorname{sim}\left(\mathrm{v}_{\mathrm{jp}}, \mathrm{v}_{\mathrm{jq}}\right)$ is larger than a pre-specified threshold, the two $t_{j p}$ and $t_{j q}$ are considered similar.

The objective of this method is to build the conditional probability of $\mathrm{p}\left(\mathrm{u}_{\mathrm{i}} \mid \mathrm{u}_{\mathrm{j}}\right)$ estimating the likelihood that user $u_{i}$ is similar to user $u_{j}$ in terms of user $u_{j}$ 's information interests. The Equation (1) is defined to calculate how similar user $u_{i}$ is interested in keyword $k$ given that user $\mathrm{u}_{\mathrm{j}}$ is interested in the keyword $\mathrm{k}$ :

$$
\mathrm{p}_{\mathrm{k}}\left(\mathrm{u}_{\mathrm{i}} \mid \mathrm{u}_{\mathrm{j}}\right)=\frac{\mathrm{n}_{\mathrm{ij}}^{\mathrm{k}}}{\mathrm{n}_{\mathrm{j}}^{\mathrm{k}}}
$$

where, $\mathrm{n}_{\mathrm{j}}^{\mathrm{k}}$ denotes the number of tags in $\mathrm{W}_{\mathrm{j}}$ that contain keyword $\mathrm{w}_{\mathrm{k}}$ and $\mathrm{n}_{\mathrm{ij}}^{\mathrm{k}}$ denotes the number of tags in $\mathrm{W}_{\mathrm{i}}$ that contain keyword $\mathrm{w}_{\mathrm{k}}$ which are similar to some tags in $\mathrm{W}_{\mathrm{j}}$ that contain keyword $w_{k}$ as well. After every keyword has been calculated, the average of the probability $\mathrm{p}_{\mathrm{k}}\left(\mathrm{u}_{\mathrm{i}} \mid \mathrm{u}_{\mathrm{j}}\right)$ is used to estimate the probability $\mathrm{p}\left(\mathrm{u}_{\mathrm{i}} \mid \mathrm{u}_{\mathrm{j}}\right)$ as in Equation (2):

$$
\mathrm{p}\left(\mathrm{u}_{\mathrm{i}} \mid \mathrm{u}_{\mathrm{j}}\right)=\sum_{\mathrm{k} \in \mathrm{W}} \mathrm{p}_{\mathrm{k}}\left(\mathrm{u}_{\mathrm{i}} \mid \mathrm{u}_{\mathrm{j}}\right) /|\mathrm{W}|
$$

where, $\mathrm{W}=\left\{\mathrm{w}_{1}, \ldots, \mathrm{W}_{\mathrm{r}}\right\}$ is the set of all keywords in $\mathrm{W}_{\mathrm{i}}$ or $\mathrm{W}_{\mathrm{j}}$. Finally, conditional probability $\mathrm{p}\left(\mathrm{u}_{\mathrm{i}} \mid \mathrm{u}_{\mathrm{j}}\right)$ is used to measure the trust from user $u_{j}$ to user $u_{i}$. Given $u_{j}$, the higher the probability of $\mathrm{p}\left(\mathrm{u}_{\mathrm{i}} \mid \mathrm{u}_{\mathrm{j}}\right)$, the higher trust that user $u_{j}$ has to $u_{i}$, since user $u_{i}$ has similar interest as $u_{j}$.

\subsection{Tidal Trust (TT)}

TT method is used to compute how much one user should trust another unknown user based on the paths that connect them in the social network as well as the trust values along those paths. The trust is then accumulated over neighbours of varying distance to create a ranked list approach. The top ranked recommended users are then presented to the user (Golbeck, 2006).

We employ TT approach to compute trust network as the second source of trust value in our proposed method. The social tagging system does not collect explicit rating about trust among the user. For example, user's like the URL bookmark by user $\mathrm{j}$, user $\mathrm{s}$ adds user $\mathrm{j}$ into his/her network. Therefore, we assume that the trust value for user $\mathrm{s}$ trust user $\mathrm{j}$ is 1 . The trust value can be computed by Equation (3):

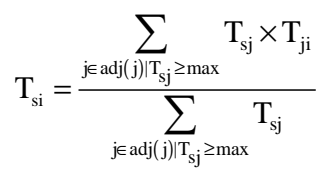

The source's inferred trust rating for the sink $\left(\mathrm{T}_{\mathrm{si}}\right)$ is a weighted average of the source's neighbours' ratings of the sink. $s$ is a source and $i$ is a sink. adj represents all the paths that connect user $s$ to user $i$ in the social network along adjacent neighbours. $\mathrm{j}$ indicates the total adjacent neighbours. It represents a few paths from user s to user i. max is the trust threshold which can be established by taking the maximum of the trust paths leading to the sink or user i. This means that each user in the process computes its trust in another user as a weighted mean and only takes into account information from users that he has rated at least as high as max.

\subsection{Hybrid User Trust Combination}

The proposed hybrid User Trust method integrates two different methods to compute the trust network. The first method is using users' interest similarity from the ST proposed by Bhuiyan et al. (2010). The second method is inferring the trust network from social network based on TT as proposed by Golbeck (2006). Having both trust values, we then sum up the trust network value of these two models to find most trusted users as 
neighbours in order to make automated recommendations. However, computation of trust network by ST or TT maybe zero, which means there is no trust exists between the users. Therefore, in order to preserve more quality neighbours and to avoid over fitting errors, the method of combination by summing up is applied. In this way, when division, multiplication or subtraction is applied, the neighbour datasets are underestimated when ST or TT trust value is zero. The algorithm of user recommendation based on hybrid User Trust method algorithm is shown below.

\section{Input:}

$\mathrm{j}$, a query user.

$\mathrm{k}$, number of neighbours to consider.

$\mathrm{n}$, number of users to recommend.

Output: Q, a set of recommended users.

//Computation trust value.

for each $\mathrm{i} € \mathrm{U}$ do//U, list of users ST

Trust $1[\mathrm{i}]=\mathrm{p}(\mathrm{i} \mid \mathrm{j}) / /$ computing the trust based on

end for

for each $\mathrm{i} € \mathrm{U} \mathrm{do} / / \mathrm{U}$, list of users TT

Trust $2[\mathrm{i}]=\mathrm{T}_{\mathrm{ji}} / /$ computing the trust based on

end for

//Combination trust value.

for each $\mathrm{i} € \mathrm{U} \mathrm{do} / / \mathrm{U}$, list of users

Trust $[i]=$ Trust $1[i]+$ Trust $2[i]$

end for

//Form the neighbourhood.

for each i $€ U$ do // U, list of users

if Trust [i] $>0 / /$ If trust $>0$, user $\mathrm{i}$ is the nearest neighbours to user $\mathrm{j}$.

$\mathrm{K} / / \mathrm{K}$ represent all the nearest neighbours to user $\mathrm{j}$. $\mathrm{K}<=\mathrm{k}$, number of neighbours to consider end if

end for

//Recommendation.

for each $\mathrm{i} € \mathrm{~K}$ do

for each $\mathrm{u}$ that $\mathrm{i}$ add $\mathrm{u}$ as network do

$\mathrm{w}_{\mathrm{u}}+=\operatorname{Trust}[\mathrm{i}] / \mathrm{k} / / \mathrm{w}_{\mathrm{u}}$ represent all the recommended user

end for

end for

Sort users by wu;

Let $\mathrm{Q}$ be the top $\mathrm{n}$ recommended users from wu; return $\mathrm{Q}$;

\subsection{Online Phase of Hybrid User Trust for User Recommendation}

We ignore users that have no trust value for the target user to form the neighbourhoods. Once the most trusted neighbours have been discovered, the set of users who are followed by most trusted neighbours will be identified by group as well as by their frequency. Using this set of users that are followed by most trusted neighbours, the $\mathrm{N}$ most frequent of the users is recommended to the target user.

\section{RESULTS AND DISCUSSION}

In order to evaluate the performance of the hybrid User Trust method, two main experiments have been conducted. The resulting top $N$ user recommendation is used to find the most trusted neighbouring users within a social tagging system. From these neighbours, a set of recommended user is constructed. The hybrid User Trust (UserTrust) method is compared with the previous methods; the user-based collaborative filtering with Pearson Correlation Coefficient (PCC) (Resnick et al., 1994), Tidal Trust (TT) (Golbeck, 2006), UserRec (Zhou et al., 2010), tag-based Similarity Trust approach (ST) (Bhuiyan et al., 2010) and incorporation of social network information in CF (PCC-SN) (Liu and Lee, 2010).

\subsection{Data Set}

The Del.icio.us dataset (http://www.delicious.com/) contains users' tagging activities, network information and fan information. The dataset consists of four tables, which are user, network, fan and tagging. The data collection lasted for one month back in the year of 2009 by the Chinese University of Hong Kong. The same dataset has also been used in Zhou et al. (2010).

In order to understand some key characteristics of our dataset, we consider Fig. 2 shows distribution of the number of users in a user's network based on Power Law distribution. Figure 3 shows the distribution of the number of fans of a user. Surprisingly, this distribution also follows Power Law distribution. We observe that the majority of users are condensed in a small fraction of the user network or fan due to no effective way for the user to discover other users with common interest.

Figure 4 demonstrates the relationship between a user's number of bookmarks and his or her number of fans, where there is a positive relationship. The reason why this happens is similar to why the Web portals become very popular and have plenty of visits every day. There-fore, we use users' interest similarity based on the tag information to develop trust network in the absence of explicit rating data to find most trusted neighbours and replace the user-based Collaborative Filtering (CF) approach where it finds the neighbours based on overlapped or common previous ratings data. 


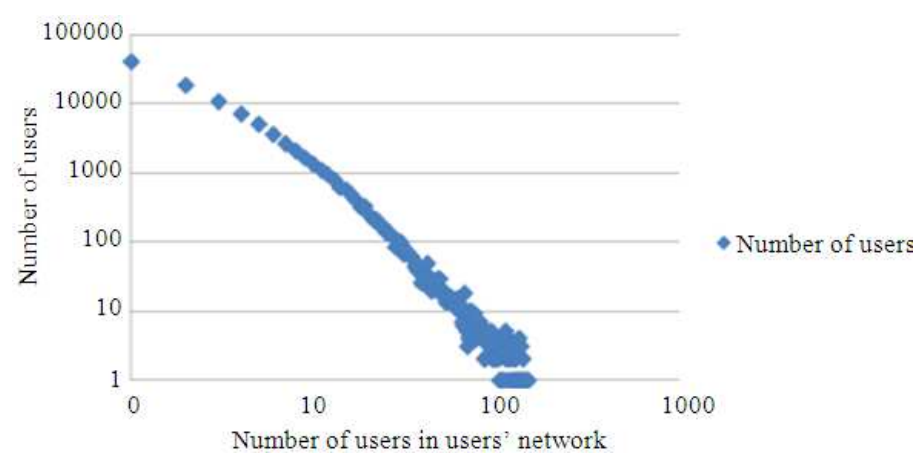

Fig. 2. Distribution of number of users in users' network

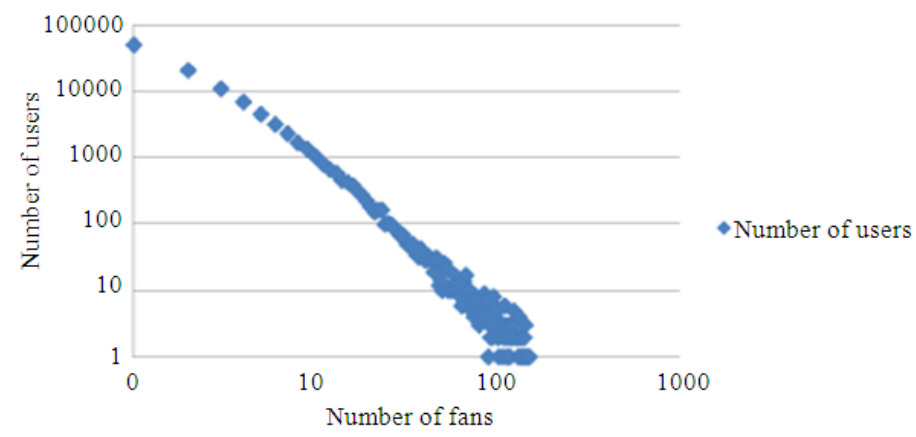

Fig. 3. Distribution of number of fans

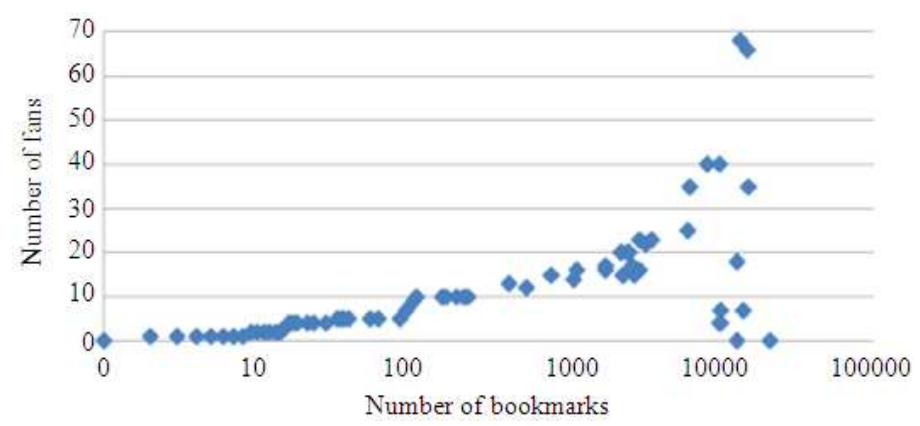

Fig. 4. Relation between number of bookmarks and number of fans

In analyzing the relationship between the user bookmarks, we only selected users with at least bookmark five URLs and the URLs are also used by at least another three users. This is to avoid sparse data and memory constraints. Besides, the selected users should also have added at least three other users in their network.

The final dataset consisted of 2,376 users, 139,707 unique tags and 1,190,762 URLs. We divided the dataset into a training set and a testing set using 80/20 percent test ratio. The training set was used to build the model while the test set was used to generate and evaluate recommendation. The users in the test set have between 3 to 10 users in their network.

\subsection{Evalution Metrics}

The recall and precision metrics are used to evaluate the performance of recommendation. The top-N users are recommended to the users. For comparison purposes, we will use recommended users $\mathrm{N}=3,5,10,15,20,30$ and 50 . Besides, we also use neighbourhood of size $\mathrm{K}=50$, $100,150,200,250$ and 300. Recall and precision for a user list as recommended to user $u_{i}$ is computed based on the Equation (4) and (5): 
Recall $=\frac{\left|T_{i} \cap P_{i}\right|}{\left|T_{i}\right|}$

Precision $=\frac{\left|T_{i} \cap P_{i}\right|}{P_{i}}$

where, $T_{i}$ is the set of all users followed by user $u_{i}$ and $P_{i}$ is the set of all recommended users generated by the recommender system. Based on the recall and precision, it can be observed that the values of recall and precision are sensitive to the size of the recommended users list. Since recall and precision are inversely correlated and are dependent on the size of the recommended user list, they must be considered together to completely evaluate the performance of a recommender system. To combine them, F1 measure is used during the evaluation, which can be computed using Equation (6):

F1 measure $=\frac{2 \times \text { Recall } \times \text { Precision }}{\text { Recall }+ \text { Pr ecision }}$

Finally, the proposed hybrid User Trust method will be compared against previous methods, which are PCC, TT, UserRec, ST and PCC-SN in order to evaluate its quality. The recall, precision and F1 measure parameters are used as the evaluation metric for the experiment on Del.icio.us dataset.

\subsection{Experimental Results}

The size of the neighbourhood has significant impact on the recommendation quality (Deshpande and Karypis, 2004; Karypis, 2001; Liu and Lee, 2010; Sarwar et al., 2000) for hybrid User Trust, PCC, TT, ST and PCC-SN. Therefore, the first experiment does not dominate the neighbourhood size. It is because to find out the unit length normalization was performed so that users that have bookmarked many URLs or added a lot user in network will not dominate the aggregate neighbourhoods. The second experiment is domination of the neighbourhood size to determine the effect of neighbourhood size.

\subsection{Experiments without Neighbourhood Size}

In the first experiment, we implemented six methods hybrid User Trust, PCC, TT, UserRec, ST and PCC-SN to recommend top-N users to each of users in the test set using different values for $\mathrm{N}=3,5,10,15,20,30$ and 50 . The hybrid User Trust, PCC, TT, ST and PCC-SN methods are used to compute the neighbourhood and the most-frequent item algorithm to generate prediction. The size of the neighbourhood has significant impact on the recommendation quality for hybrid User Trust, PCC, TT, ST and PCC-SN techniques. The UserRec is a tag-graph based community detection method to model the user's personal interests, which are further represented by discrete topic distributions. The similarity values between user's topic distributions are measured by Kullback Leibler divergence (KL-divergence) and the similarity values are further used to perform interest-based user recommendation. Therefore, the recommendation accuracy for UserRec is not influenced by the neighbourhood size. We did not dominate the neighbourhood size in these experiments. It is because to find out the unit length normalization was performed so that users that have bookmarked many URLs or added a lot user in network will not dominate the aggregate neighbourhoods.

We executed the experiment using the training data and used the test set to compute recall, precision and F1 measure. The results are shown in Fig. 5-7 respectively.

Based on Fig. 5-7, it can be observed that among the three evaluation metrics, the proposed hybrid User Trust method achieved the best result among all the six methods. This is due to the incorporation of tags and social network information in $\mathrm{CF}$ for finding the neighbours can reduce trustworthiness attack by malicious users into the recommender system. The three methods UserRec, TT and hybrid User Trust performed closely but hybrid User Trust is slightly better than these three because UserRec only uses tags to calculate similarity of users and TT constructs the trustworthiness by social network. Therefore, UserRec and TT face attack by malicious users' problem. Among all, the proposed hybrid User Trust performed better than the PCC, PCC-SN and ST methods. PCC-SN performed significantly better than PCC and ST methods. This is due to incorporating social network information in CF to find the neighbours which source from people whom know or trust. Therefore, we know that the social network information is better than tags to construct the trustworthiness. ST performed significantly better than PCC method because it reduces the semantic meaning problem for the tags and solves the disadvantage of PCC which overemphasizes the similarities of user' opinions. The PCC, TT, PCC-SN, ST and hybrid User Trust also used the same recommendations methods with different means in finding the neighbours.

From Fig. 5 and 6 the recommendation performances improve when the methods recommends top- 3 to top-10 users to each of users in the test set. After the top-10 value, the increase rate of top- $\mathrm{N}$ diminishes and the curve tends to be flat for hybrid User Trust due to over-fitting errors caused by not dominating neighbourhood size. 
Norwati Mustapha et al. / Journal of Computer Science 9 (8): 1008-1018, 2013

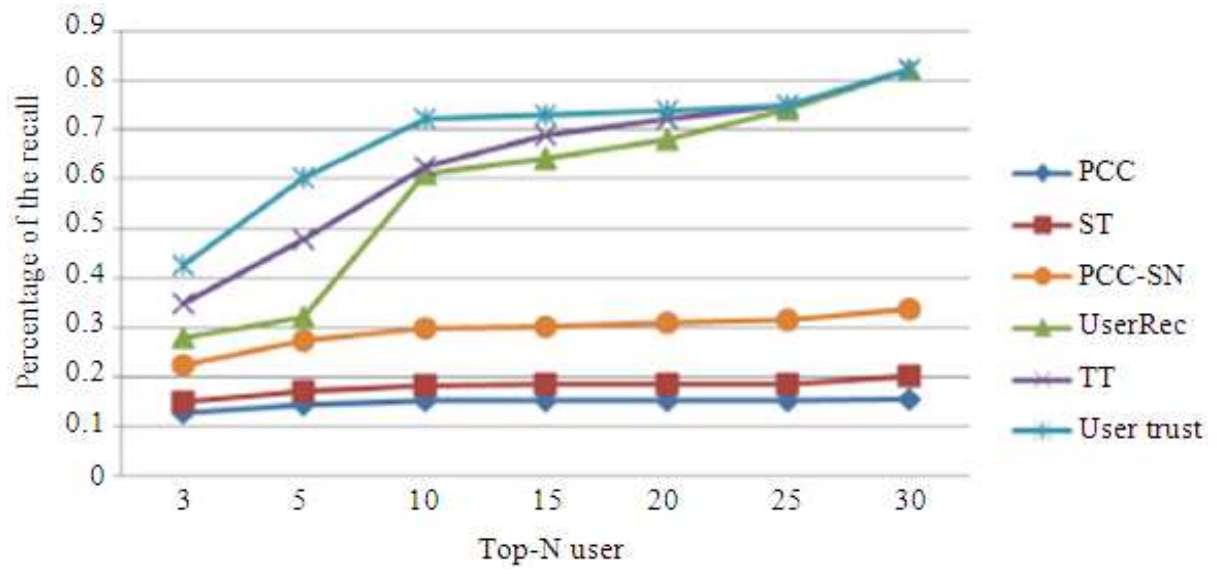

Fig. 5. Evaluation top-N users with recall metric

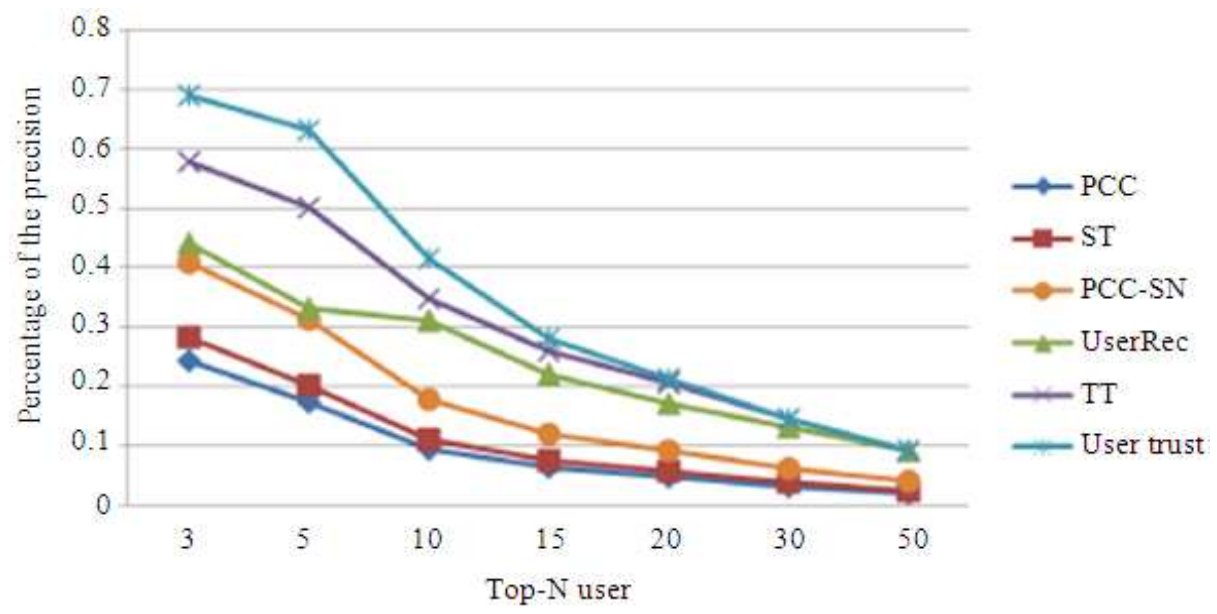

Fig. 6. Evaluation top-N users with precision metric

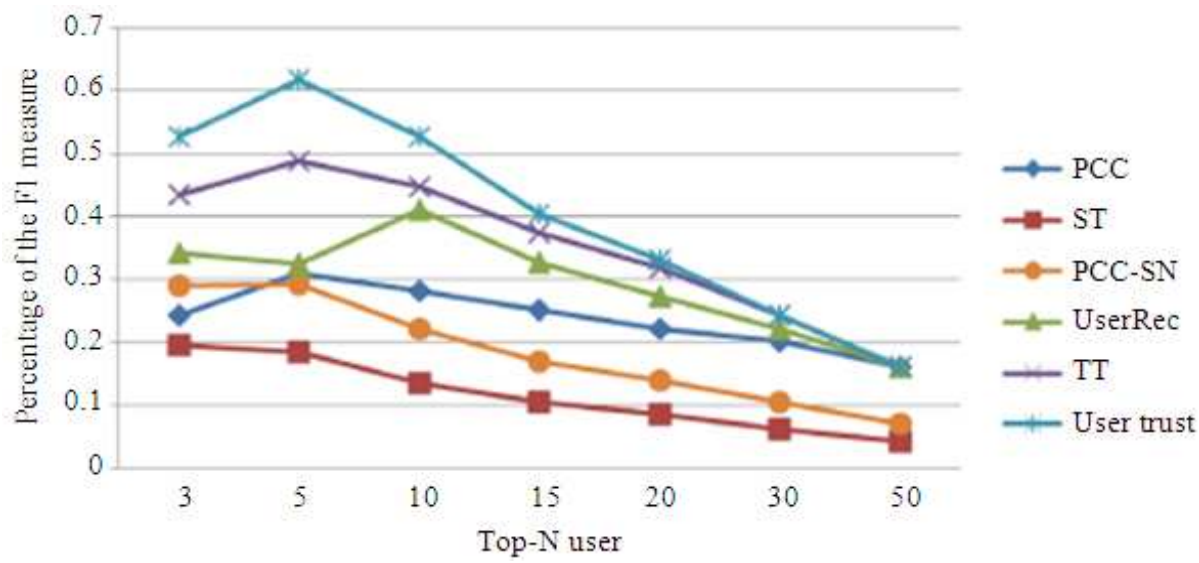

Fig. 7. Evaluation top-N users with F1 measure metric 
Norwati Mustapha et al. / Journal of Computer Science 9 (8): 1008-1018, 2013

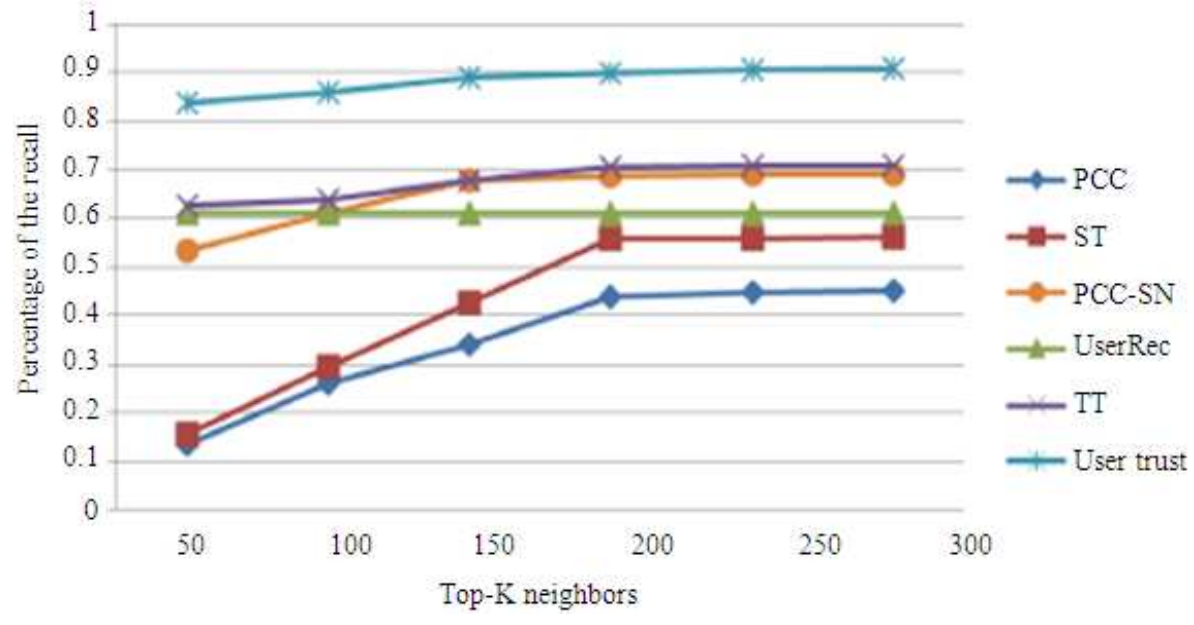

Fig. 8. Evaluation neighbourhood size with recall metric

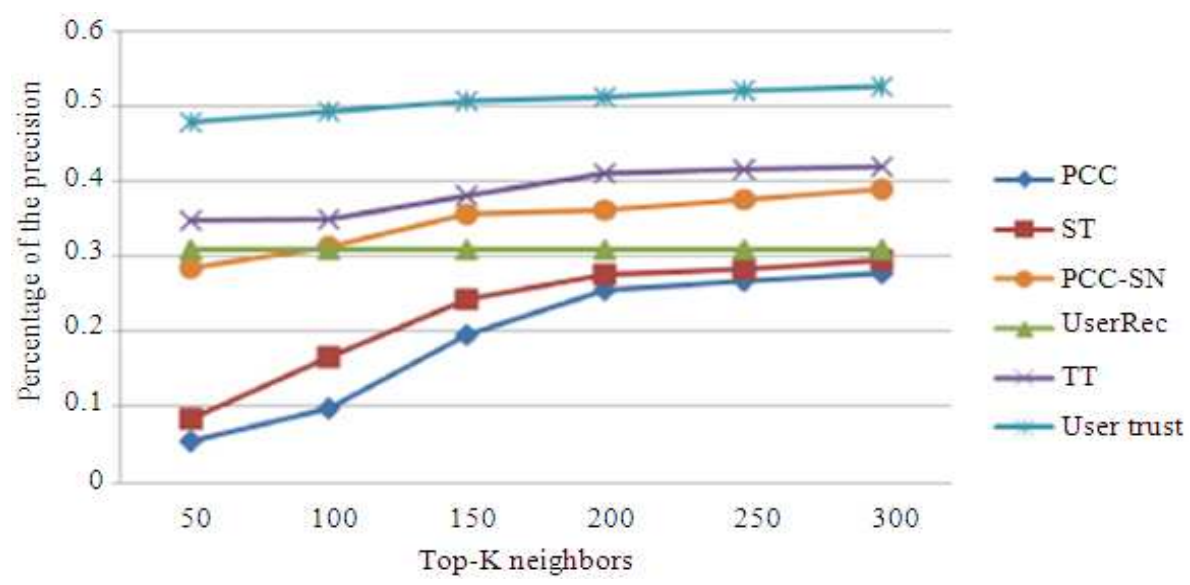

Fig. 9. Evaluation neighbourhood size with precision metric

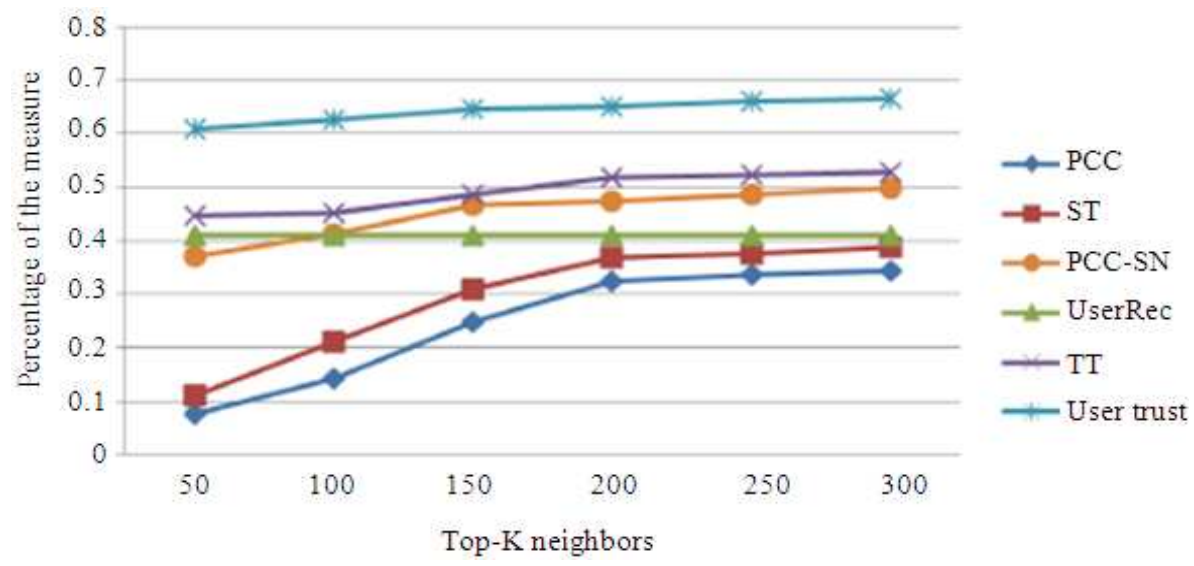

Fig. 10. Evaluation neighbourhood size with F1-meausre metric 
In general, the quality may increase as we increase the number of neighbours. However, after a certain point, the improvement gains diminishment and the quality becomes worse due to over-fitting errors. Therefore, we select 10 as the optimal number of top-N recommendation. We are able to observe vast differences between hybrid User Trust, PCC, TT, UserRec, ST and PCC-SN methods.

\subsection{Experiments with Neighbourhood Size}

The size of the neighbourhood is known to have a significant impact on the quality of recommendation. To determine the sensitivity of this parameter, we performed an experiment where we varied the number of neighbours $\mathrm{K}=50,100,150,200,250$ and 300 in calculating the recall, precision and F1 measure. Based on findings presented in Section 3.4, we fixed the number of recommendation to 10 as the optimal top-N recommendation because we are able to observe vast differences between hybrid User Trust, PCC, TT, UserRec, ST and PCC-SN methods. The results are shown in Fig. 8-10.

As we can see from Fig. 8-10, the size of the neighbourhood does affect the quality of top-10 recommendations for hybrid User Trust, PCC, ST, TT and PCC-SN methods. The same figures also show that the quality of recommendation increases from the number of neighbours' ranging between 50 to 200 . However, after number of neighbours reached 200, the improvement gains diminish and the curve tends to be flat. It is be-cause selecting too many neighbours who are not very similar to the target user to make recommendation hence affect accuracy. Therefore, we select 200 as our optimal number of nearest neighbours because it is large enough to capture all the latent relationships in the matrix yet small enough to avoid over-fitting errors.

\section{CONCLUSION}

We have presented a hybrid User Trust method for user recommendation approach which allows users to find other users with similar interest in social tagging system. Our experiment result shows that the proposed hybrid User Trust method outperforms the PCC, TT, UserRec, ST and PCC-SN.

There are some aspects that could be improved in our hybrid User Trust method. One is involving the investigating of alternative hybrid user recommenders. New recommenders that cover other informational channels will be considered and alternative methods for hybridizing recommenders will be explored. Two, we are also interested in incorporating methods in natural language processing and semantic analysis for overcoming the problems of tag ambiguity, in effort to improve the quality of the recommendation. Finally, we would like to study the impact of hybrid User Trust when dealing with application domains other than the social tagging systems, for example in movies, CDs, fashion and so forth. The results obtained may differ, owing to distinct characteristics concerning the structure of genre classification inherent to these domains.

\section{REFERENCES}

Begelman, G., P. Keller and F. Smadja, 2006. Automated tag clustering: Improving search and exploration in the tag space. Technion-Israel Institute of Technology.

Bennett, J. and S. Lanning, 2007. The netflix prize. Proceedings of the KDD Cup and Workshop, Aug. 1212, ACM Press, San Jose, California, USA., pp: 3-6.

Bhuiyan, T., Y. Xu, A. Jøsang, H. Liang and C. Cox, 2010. Developing trust networks based on user tagging information for recommendation making. Proceedings of the 11th International Conference on Web Information Systems Engineering, Dec. 12-14, Springer Berlin Heidelberg, Hong Kong, China, pp: 357-364. DOI: 10.1007/978-3-642-17616-6_32

Deshpande, M. and G. Karypis, 2004. Item-based top- $n$ recommendation algorithms. ACM Trans. Inform. Syst., 22: 143-177. DOI: 10.1145/963770.963776

Gemmell, J., T. Schimoler, M. Ramezani, L. Christiansen and B. Mobasher, 2009a. Improving folkrank with item-based collaborative filtering. Proceedings of the Workshop on Recommender Systems and the Social Web, Oct. 22-25, ACM Press, New York, pp: 17-24.

Gemmell, J., M. Ramezani, T. Schimoler, L. Christiansen and B. Mobasher, 2009b. The impact of ambiguity and redundancy on tag recommendation in folksonomies. Proceedings of the 3rd ACM Conference on Recommender Systems, Oct. 23-25, ACM Press, New York, USA., pp: 45-52. DOI: 10.1145/1639714.1639724

Golbeck, J., 2006. Generating predictive movie recommendations from trust in social networks. Proceedings of the 4th International Conference on Trust Management, May 16-19, Springer Berlin Heidelberg, Pisa, Italy, pp: 93-104. DOI: 10.1007/11755593_8 
Hotho, A., R. Jaschke, C. Schmitz and G. Stumme, 2006. Information retrieval in folksonomies: Search and ranking. Proceedings of the 3rd European Conference on the Semantic Web: Research and Applications, Jun. 11-14, Springer Berlin Heidelberg, Budva, Montenegro, pp: 411-426. DOI: 10.1007/11762256_31

Jaschke, R., L. Marinho, A. Hotho, L. Schmidt-Thieme and G. Stumme, 2007. Tag recommendations in folksonomies. Proceedings of the 11th European Conference on Principles and Practice of Knowledge Discovery in Databases, Sept. 17-21, Springer Berlin Heidelberg, Warsaw, Poland, pp: 506-514. DOI: 10.1007/978-3-540-74976-9_52

Karypis, G., 2001. Evaluation of item-based top-n recommendation algorithms. Proceedings of the 10th International Conference on Information and Knowledge Management, Nov. 06-11, ACM Press, New York, USA., pp: 247-254. DOI: 10.1145/502585.502627

Konstan, J.A., B.N. Miller, D. Maltz, J.L. Herlocker and L.R. Gordon et al., 1997. GroupLens: Applying collaborative filtering to Usenet news. Commun. ACM., 40: 77-87. DOI: 10.1145/245108.245126

Linden, G., B. Smith and J. York, 2003. Amazon.com recommendations: Item-to-item collaborative filtering. IEEE Internet Comput., 7: 76-80. DOI: 10.1109/MIC.2003.1167344

Liu, F. and H.J. Lee, 2010. Use of social network information to enhance collaborative filtering performance. Expert Syst. Applic., 37: 4772-4778. DOI: 10.1016/j.eswa.2009.12.061

Noll, M.G. and C. Meinel, 2007. Web search personalization via social bookmarking and tagging. Proceedings of the 6th international The semantic Web, Nov. 11-15, Springer Berlin Heidelberg, Busan, Korea, pp: 367-380. DOI: 10.1007/978-3540-76298-0_27

Resnick, P., I. Neophytos, S. Mitesh, P. Bergstrom and J. Riedl, 1994. GroupLens: An open architecture for collaborative filtering of netnews. Proceedings of the ACM Conference on Computer Supported Cooperative Work, ACM Press, New York, USA., pp: 175-186.
Sarwar, B., G. Karvpis, J. Konstan and J. Riedl, 2000. Analysis of recommendation algorithms for ecommerce. Proceedings of the 2nd ACM Conference on Electronic Commerce, Oct. 17-20, ACM Press, New York, USA., pp: 158-167. DOI: 10.1145/192844.192905

Shepitsen, A., J. Gemmell, B. Mobasher and R. Burke, 2008. Personalized recommendation in social tagging systems using hierarchical clustering. Proceedings of the ACM Conference on Recommender Systems, Oct. 23-25, ACM Press, New York, USA., pp: 256-266. DOI: 10.1145/1454008.1454048

Tso-Sutter, K.H.L., L.B. Marinho and L. SchmidtThieme, 2008. Tag-aware recommender systems by fusion of collaborative filtering algorithms. Proceedings of the ACM Symposium on Applied Computing, Mar. 16-20, ACM Press, New York, USA., pp: 1995-1999. DOI: 10.1145/1363686.1364171

Tyler, S.K. and Y. Zhang, 2008. Open domain recommendation: Social networks and collaborative filtering. Proceedings of the 4th International Conference on Advanced Data Mining and Applications, Oct. 8-10, Springer Berlin Heidelberg, Chengdu, China, pp: 330-341. DOI: 10.1007/978-3540-88192-6_31

Yubo, J., C. Hao and C. Huang, 2010. A collaborative filtering recommendation algorithm based on user trust model. Proceedings of the 1st International Conference on Networking and Distributed Computing, Oct. 21-24, IEEE Xplore Press, Hangzhou, pp: 213-217. DOI: 10.1109/ICNDC.2010.51

Zhou, C.T., H. Ma, R.M. Lyu and I. King, 2010. UserRec: A user recommendation framework in social tagging systems. Proceedings of the 24rth AAAI Conference on Artificial Intelligence, (AAAI' 10), Association for the Advancement of Artificial Intelligence, pp: 1486-1491. 